\title{
Low-loss metasurface optics down to the deep ultraviolet region
}

\author{
Cheng Zhang ${ }^{1,2,3}$, Shawn Divitt ${ }^{2,3}$, Qingbin Fan ${ }^{4}$, Wenqi Zhu ${ }^{2,3}$, Amit Agrawal $\mathbb{B}^{2,3}$, Yanqing Lu ${ }^{4}$, Ting Xu ${ }^{4}$ and
} Henri J. Lezec ${ }^{2}$

\begin{abstract}
Shrinking conventional optical systems to chip-scale dimensions will benefit custom applications in imaging, displaying, sensing, spectroscopy, and metrology. Towards this goal, metasurfaces_planar arrays of subwavelength electromagnetic structures that collectively mimic the functionality of thicker conventional optical elements-have been exploited at frequencies ranging from the microwave range up to the visible range. Here, we demonstrate highperformance metasurface optical components that operate at ultraviolet wavelengths, including wavelengths down to the record-short deep ultraviolet range, and perform representative wavefront shaping functions, namely, highnumerical-aperture lensing, accelerating beam generation, and hologram projection. The constituent nanostructured elements of the metasurfaces are formed of hafnium oxide-a loss-less, high-refractive-index dielectric material deposited using low-temperature atomic layer deposition and patterned using high-aspect-ratio Damascene lithography. This study opens the way towards low-form factor, multifunctional ultraviolet nanophotonic platforms based on flat optical components, enabling diverse applications including lithography, imaging, spectroscopy, and quantum information processing.
\end{abstract}

\section{Introduction}

An optical metasurface is a planar array of subwavelength electromagnetic structures that emulate the operation of a conventional refractive, birefringent, or diffractive optical component, such as a lens, waveplate, or hologram, through individually tailored amplitude, phase, or polarization transformations of the incident light ${ }^{1-9}$. Dielectric materials such as amorphous $\mathrm{Si}^{10,11}$, polycrystalline $\mathrm{Si}^{12}$, titanium dioxide $\left(\mathrm{TiO}_{2}\right)^{13,14}$, and gallium nitride $(\mathrm{GaN})^{15,16}$ have been used to realize metasurfaces operating at infrared and visible frequencies. The scarcity of dielectric materials that are characterized

\footnotetext{
Correspondence: Cheng Zhang (cheng.zhang@hust.edu.cn) or

Ting Xu (xuting@nju.edu.cn) or Henri J. Lezec (henri.lezec@nist.gov)

'School of Optical and Electronic Information \& Wuhan National Laboratory for Optoelectronics, Huazhong University of Science and Technology, 430074

Wuhan, China

${ }^{2}$ Physical Measurement Laboratory, National Institute of Standards and

Technology, 20899 Gaithersburg, MD, USA

Full list of author information is available at the end of the article

These authors contributed equally: Cheng Zhang, Shawn Divitt, Qingbin Fan
}

by low optical loss at higher frequencies and simultaneously amenable to high-aspect-ratio nanopatterning has impeded the development of metasurfaces for applications in the ultraviolet (UV) range, a technologically important spectral regime hosting diverse applications in lithography, imaging, spectroscopy, time keeping, and quantum information processing ${ }^{17-19}$. To date, metasurfaces designed for operation in the near-UV range (UV-A; free-space wavelength range: $315 \mathrm{~nm} \leq \lambda_{0} \leq$ $380 \mathrm{~nm}$; energy range: $3.26 \mathrm{eV} \leq E_{0} \leq 3.94 \mathrm{eV}$ ) have been implemented using niobium pentoxide $\left(\mathrm{Nb}_{2} \mathrm{O}_{5}\right)$, down to an operation free-space wavelength of $\lambda_{0}=355 \mathrm{~nm}^{20}$. Crystalline $\mathrm{Si}$ has been used to realize metasurfaces operating down to $\lambda_{0}=290 \mathrm{~nm}^{21}$, a wavelength that falls within the mid-UV range (UV-B; $280 \mathrm{~nm} \leq \lambda_{0} \leq 315 \mathrm{~nm}$; $3.94 \mathrm{eV} \leq E_{0} \leq 4.43 \mathrm{eV}$ ), but the device efficiencies remain limited by the severe absorption loss associated with illumination frequencies above the bandgap of $\mathrm{Si}\left(E_{\mathrm{g}} \approx\right.$ $1.1 \mathrm{eV})$. In both studies, the demonstrated functionalities are limited to hologram generation and beam deflection,

\section{(c) The Author(s) 2020}

(c) (i) Open Access This article is licensed under a Creative Commons Attribution 4.0 International License, which permits use, sharing, adaptation, distribution and reproduction c. in any medium or format, as long as you give appropriate credit to the original author(s) and the source, provide a link to the Creative Commons license, and indicate if changes were made. The images or other third party material in this article are included in the article's Creative Commons license, unless indicated otherwise in a credit line to the material. If material is not included in the article's Creative Commons license and your intended use is not permitted by statutory regulation or exceeds the permitted use, you will need to obtain permission directly from the copyright holder. To view a copy of this license, visit http://creativecommons.org/licenses/by/4.0/. 
while other important wavefront shaping functionalities that can be empowered by optical metasurfaces, such as high-numerical-aperture focusing and structured beam generation, have not yet been achieved. Meanwhile, metasurfaces that can operate at even higher frequencies, such as within the deep-UV range (longer wavelength portion of UV-C; $190 \mathrm{~nm} \leq \lambda_{0} \leq 280 \mathrm{~nm} ; 4.43 \mathrm{eV} \leq E_{0} \leq$ $6.53 \mathrm{eV}$ ), have not been realized due to the challenge of identifying a dielectric material that has a suitably low optical absorption coefficient in that range and can be patterned into high-aspect-ratio nanostructures using the available nanofabrication techniques.

Here, we report high-performance dielectric metasurfaces that operate over a broad UV range, including within the record-short, deep-UV regime, and perform representative wavefront shaping functionalities. The constituent nanostructured elements of the metasurfaces are formed of hafnium oxide $\left(\mathrm{HfO}_{2}\right)_{-}$-a UV-transparent, high-refractive-index dielectric material. Although $\mathrm{HfO}_{2}$ has been commonly exploited as a high-static-dielectricconstant (high- $\kappa$ ) material in integrated circuit fabrication $^{22,23}$, its applications in photonics have largely been limited to optical coatings based on planar thin films, in particular due to the difficulty of patterning the material into high-aspect-ratio nanostructures. In this work, we overcome this limitation and use $\mathrm{HfO}_{2}$, for the first time, to implement meta-devices operating in the UV and deepUV regimes. We deposit high-quality, UV-transparent $\mathrm{HfO}_{2}$ films using low-temperature atomic layer deposition (ALD) and pattern the films using a high-aspect ratio, resist-based Damascene lithography technique ${ }^{24-26}$. We implement metasurfaces designed for operation at three representative UV wavelengths of 364, 325, and $266 \mathrm{~nm}$, which perform a variety of optical functions, namely, high-numerical-aperture lensing, accelerating beam generation, and hologram projection, including under spin control for the last two applications. This achievement opens the way for low-form-factor and multifunctional photonic systems based on UV flat optics, and suggests promising applications in photolithography, highresolution imaging, UV spectroscopy and quantum information processing.

\section{Results}

\section{Material choice and fabrication approach}

The implemented metasurface devices consist of $\mathrm{HfO}_{2}$ nanopillars of either circular or elliptical in-plane crosssections (Fig. 1a), densely arrayed on a transparent UVgrade fused silica substrate with a low refractive index (Supplementary Information, Fig. S1). The choice of $\mathrm{HfO}_{2}$ - a material most commonly exploited for its high static dielectric constant as a transistor gate insulator in complementary metal oxide semiconductor (CMOS) integrated circuits-is guided by the promise of both a large refractive index $\left(n>2.1\right.$ for $\left.\lambda_{0}<400 \mathrm{~nm}\right)$ and a wide bandgap $E_{\mathrm{g}}=5.7 \mathrm{eV}\left(\lambda_{\mathrm{g}}=217 \mathrm{~nm}\right)$ located well within the deep-UV range, leading to a negligible extinction coefficient $(k \approx 0)$ for $\lambda_{0} \geq \lambda_{\mathrm{g}}$. Although the requirement of nanopillar dimensions with a wavelength-scale height (several hundred nanometers), a subwavelength-scale inplane circle diameter or ellipse minor axis (few tens of nanometers), and vertical sidewalls suggest that pattern transfer with a directional dry-etching technique such as reactive ion etching would be optimal, we were unable to identify a suitable high-aspect-ratio, dry-etching chemistry for $\mathrm{HfO}_{2}$ (a material commonly patterned by nondirectional wet chemical etching ${ }^{27}$ ). We instead explore the use of Damascene lithography ${ }^{24-26}$ for $\mathrm{HfO}_{2}$ metasurface fabrication, a process that involves first patterning resist using electron beam (e-beam) lithography, conformally filling the open volumes of the resist template with $\mathrm{HfO}_{2}$ using $\mathrm{ALD}$, back-etching the over-coated $\mathrm{HfO}_{2}$ layer using argon (Ar) ion milling, and finally removing the remaining resist with solvent to form the required highaspect-ratio nanopillars (see "Materials and methods").

The preservation of the physical integrity of the resist template requires the use of a plasma-free thermal ALD process with a process temperature, $T_{\mathrm{p}}$, lower than the glass transition temperature (reflow temperature) of the utilized resist, $T_{\mathrm{g}}$, along with a process chemistry involving by-products that are not corrosive to the resist. Fulfilling both process tolerance requirements rules out the use of common $\mathrm{Hf}$ precursors such as Tetrakis (ethylmethylamino)Hafnium (TEMAH) ${ }^{28}$, for which the minimum $T_{\mathrm{p}}\left(\approx 150^{\circ} \mathrm{C}\right)$ is significantly higher than the $T_{\mathrm{g}}$ of common e-beam resists, or hafnium chloride $\left(\mathrm{HfCl}_{4}\right)^{29}$, for which the reaction by-product $(\mathrm{HCl})$ attacks the resist. Instead, we investigate Tetrakis(dimethylamino)Hafnium $(\mathrm{TDMAH})^{30}$ as an alternative Hf precursor for thermal ALD of high-optical-quality $\mathrm{HfO}_{2}$, using a $T_{\mathrm{p}}$ below the $T_{\mathrm{g}}$ of common e-beam organic resists (such as ZEP, for which $T_{\mathrm{g}} \approx 105^{\circ} \mathrm{C}$ ). To avoid the risk of incomplete reaction cycles and the physical condensation of precursors associated with low-temperature ALD (yielding films with defects and voids, and hence, degraded subbandgap optical properties, such as a reduced refractive index $n$ and finite extinction coefficient $k$ ), an existing ALD process which uses TDMAH and $\mathrm{H}_{2} \mathrm{O}$ precursors and operates at $T_{\mathrm{p}}=200^{\circ} \mathrm{C}$ is modified (Fig. 1b) by (1) decreasing the process temperature to $T_{\mathrm{p}}=95^{\circ} \mathrm{C}$; (2) increasing the TDMAH pulsing time, $t_{1}$, from 0.25 to $1 \mathrm{~s}$ to enable a complete reaction with the $\mathrm{OH}$ monolayer resulting from the previous cycle; and (3) increasing the $\mathrm{N}_{2}$ purging times, $t_{2}$ and $t_{4}$, from 12 to $75 \mathrm{~s}$ to ensure full removal of the excessive precursors and reaction byproducts (see "Materials and methods"). As revealed by X-ray diffraction characterization (Supplementary Information, Section II) and spectroscopic ellipsometry measurements 
a
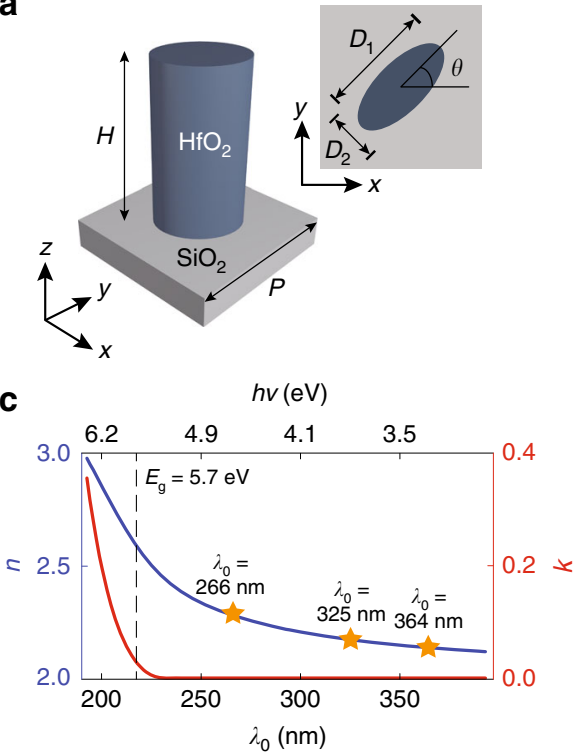

b
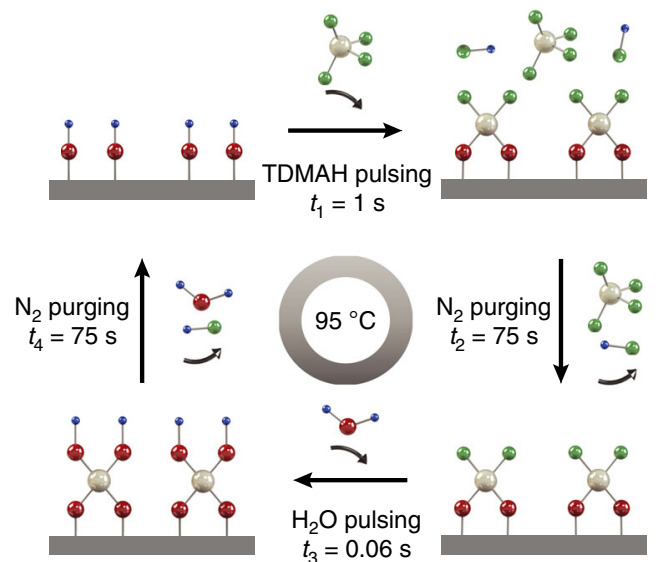

$\mathrm{Hf}$<smiles>N[Co]</smiles>

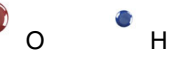

d
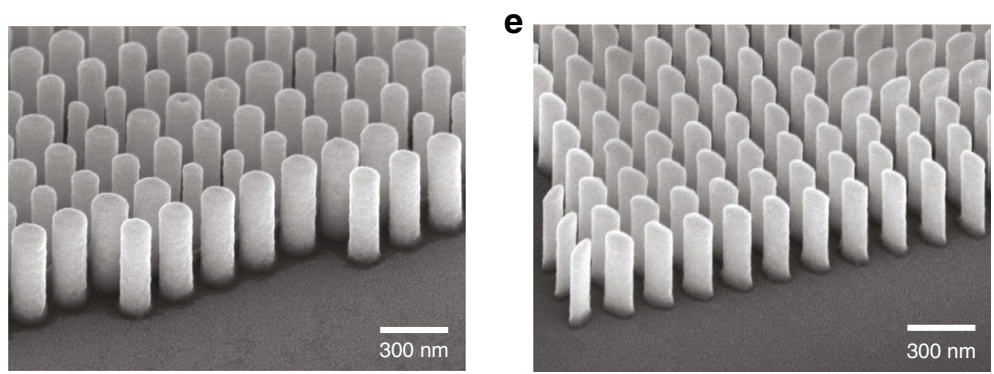

f

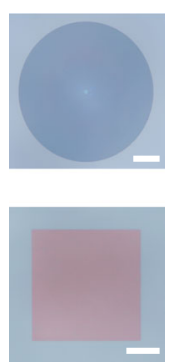

Fig. 1 Implementation of ultraviolet metasurfaces. a Schematic representation of a metasurface unit cell, consisting of a high-aspect-ratio $\mathrm{HfO}_{2}$

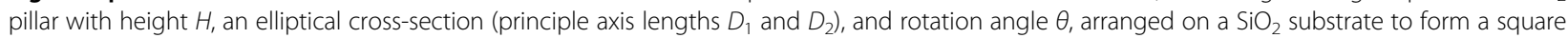
lattice with a subwavelength lattice spacing $P$. Specific optical functions are implemented via the variation in $D_{1}, D_{2}$, and $\theta$ as a function of the nanopillar position within the lattice. $\mathbf{b}$ Schematic representation of the developed low-temperature ALD cycle using the TDMAH precursor, $\mathrm{H}_{2} \mathrm{O}$ reactant, and a process temperature of $T_{\mathrm{p}}=95^{\circ} \mathrm{C}$. c Refractive index $n$ and extinction coefficient $k$ of the as-deposited $\mathrm{HfO}_{2}$ film, measured using spectroscopic ellipsometry. The values of $n$ at the three operation wavelengths targeted in this study are denoted by yellow stars. The dashed line indicates the position of the $\mathrm{HfO}_{2}$ bandgap $E_{\mathrm{g}}$. $\mathbf{d}$ Scanning electron microscopy (SEM) image of the details of a fabricated polarization-independent metalens designed for operation at $\lambda_{0}=325 \mathrm{~nm}$, showing a lattice of $500 \mathrm{~nm}$ tall, circularly shaped $\mathrm{HfO}_{2}$ nanopillars with various diameters. The viewing angle is $52^{\circ}$. e SEM image of the details of a fabricated spin-multiplexed metahologram designed for operation at $\lambda_{0}=266 \mathrm{~nm}$, showing a lattice of $480 \mathrm{~nm}$ tall, elliptically shaped $\mathrm{HfO}_{2}$ nanopillars with various in-plane cross-sections and rotation angles. The viewing angle is $52^{\circ}$. The nanopillars are coated with a layer of Au/Pd alloy $(\approx 5 \mathrm{~nm}$ thick) to suppress charging during SEM imaging. f Optical micrographs of the full metalens (top panel) and spin-multiplexed metahologram (bottom panel) corresponding to the metasurfaces described in d, e, respectively. Scale bars: $100 \mu \mathrm{m}$

(Fig. 1c and Supplementary Information, Section III), $\mathrm{HfO}_{2}$ films deposited using the modified low-temperature ALD process are amorphous and characterized by a high refractive index $(n>2.1)$ and negligible optical loss $(k \approx 0)$ over a UV wavelength interval $220 \mathrm{~nm} \leq \lambda_{0} \leq 380 \mathrm{~nm}$ spanning the full mid-UV and near-UV ranges and more than half of the deep-UV range. The measured wavelength dependences of $n$ and $k$ closely match those of a film grown using the $200^{\circ} \mathrm{C}$ reference ALD process (Supplementary Information, Fig. S4), demonstrating that the optical quality of the deposited $\mathrm{HfO}_{2}$ can be maintained at significantly lower ALD process temperatures with a suitable Hf precursor and a proper adjustment of the pulsing and purging times. Note that the $95^{\circ} \mathrm{C}$-ALDdeposited $\mathrm{HfO}_{2}$ films exhibit a high refractive index $(n>$ 2.0) and zero optical loss $(k=0)$ in the visible range $\left(380 \mathrm{~nm} \leq \lambda_{0} \leq 800 \mathrm{~nm}\right)$, making the films suitable for the fabrication of low-loss metasurface devices in this wavelength range as well (Supplementary Information, Fig. S5).

Using ZEP resist for e-beam lithography and the lowtemperature TDMAH-based ALD process for the $\mathrm{HfO}_{2}$ deposition, the proposed Damascene fabrication process is applied to yield defect-free metasurfaces, each consisting of a large array of densely packed $\mathrm{HfO}_{2}$ nanopillars on a UV-grade fused silica substrate (Fig. 1f). The nanopillars have uniform heights, circular (Fig. 1d), or elliptical (Fig. 1e) 
in-plane cross sections, and are characterized by straight, vertical, and smooth sidewalls (Fig. 1d, e, and Supplementary Information, Figs. S7-S11). The nanopillar rotation angle and two principle axis lengths in the plane of the metasurfaces $\left(\theta, D_{1}\right.$, and $D_{2}$, respectively, where $\theta=0$ and $D_{1}=D_{2}=D$ in the case of a circular crosssection) vary as a function of the nanopillar position (with $0 \leq \theta<\pi$ and $\left.50 \mathrm{~nm} \leq\left(D_{1}, D_{2}\right) \leq 160 \mathrm{~nm}\right)$ depending on the optical function implemented by the metasurface. The nanopillar height $H$ varies depending on the operation wavelength of the metasurface $(400 \mathrm{~nm} \leq H \leq 550 \mathrm{~nm})$.

We first demonstrate lenses, self-accelerating beam generators, and holograms based on polarizationindependent metasurfaces consisting of nanopillars with in-plane circular cross-sections, that operate at near-UV wavelengths of 364 and $325 \mathrm{~nm}$ (corresponding to emission lines of argon-ion and helium-cadmium lasers, respectively) with efficiencies up to $72 \%$. Further exploiting the high patterning fidelity of the Damascene technique and leveraging the negligible optical loss of the as-deposited $\mathrm{HfO}_{2}$ dielectric material across most of the UV regime, we scale down the metasurface critical dimensions to realize polarization-independent holograms operating at a deep-UV wavelength of $266 \mathrm{~nm}$ (corresponding to the emission line of an optical parametric oscillator pumped by a nanosecond Q-switched Nd:YAG laser), with relatively high efficiencies ( $>60 \%)$. Finally, by opening up the design space with the three degrees of freedom provided by elliptically shaped nanopillars $\left(\theta, D_{1}\right.$, and $\left.D_{2}\right)$, compared to the single degree of freedom provided by circularly shaped nanopillars $(D)$, we realize spin-multiplexed metasurfaces that impart independent phase shift profiles onto light emerging from the device under illumination with left-handed circularly polarized (LCP) or right-handed circularly polarized (RCP) light. The implemented self-accelerating beam generators and spin-multiplexed metaholograms operate at UV wavelengths of 364 and $266 \mathrm{~nm}$, respectively, with efficiencies up to $61 \%$.

\section{Polarization-independent UV metasurfaces}

Each polarization-independent metasurface implemented in this study (lens, self-accelerating beam generator, and hologram) consists of a square lattice of $\mathrm{HfO}_{2}$ cylindrical nanopillars, where the diameter of each pillar varies as a function of its position within the lattice. Each nanopillar acts as a truncated dielectric waveguide with top and bottom interfaces of low reflectivity, through which light propagates with a transmittance and phase shift controlled by the pillar height $H$, pillar diameter $D$, and lattice spacing $P$. For each targeted operation wavelength $\left(\lambda_{0}=364,325\right.$, and $266 \mathrm{~nm})$, a corresponding pillar height $(H=550,500$, and $400 \mathrm{~nm}$, respectively) and subwavelength lattice spacing $(P=200,190$, and $150 \mathrm{~nm}$, respectively) are chosen, along with a range of pillar diameters that yield phase shifts varying over a full range of $2 \pi$, while maintaining a relatively high and constant transmittance $([50,160 \mathrm{~nm}],[50,150 \mathrm{~nm}]$, and $[50,110 \mathrm{~nm}]$, respectively). The detailed design procedure is elaborated in Supplementary Information, Section VIII.

As a first demonstration of polarization-independent UV metasurfaces, two 500- $\mu \mathrm{m}$-diameter, polarizationindependent metalens designs, $\mathrm{L}_{364}$ and $\mathrm{L}_{325}$, with an identical numerical aperture of $\mathrm{NA}=0.6$ (corresponding to a focal length of $f=330 \mu \mathrm{m}$ ), are implemented to focus UV light at respective free-space wavelengths of $\lambda_{0}=364$ and $325 \mathrm{~nm}$ (Fig. 2a). Singlet-mode focusing of a plane wave can be achieved by implementing the radially symmetric phase shift function $\phi^{\mathrm{L}}\left(x, y, \lambda_{0}\right)=\bmod \left(\left(2 \pi / \lambda_{0}\right)(f-\right.$ $\left.\left.\sqrt{x^{2}+y^{2}+f^{2}}\right), 2 \pi\right)$, where $f$ is the focal distance normal to the plane of the lens (along the $z$ direction), $x$ and $y$ are in-plane distances along orthogonal directions from the center of the lens, and normal incidence is assumed. Each measured intensity distribution at the metalens focal plane (Supplementary Information, Section IX) reveals a circularly symmetric focal spot, characterized by a cross-section that closely matches the intensity distribution theoretically predicted for a diffraction-limited lens with a numerical

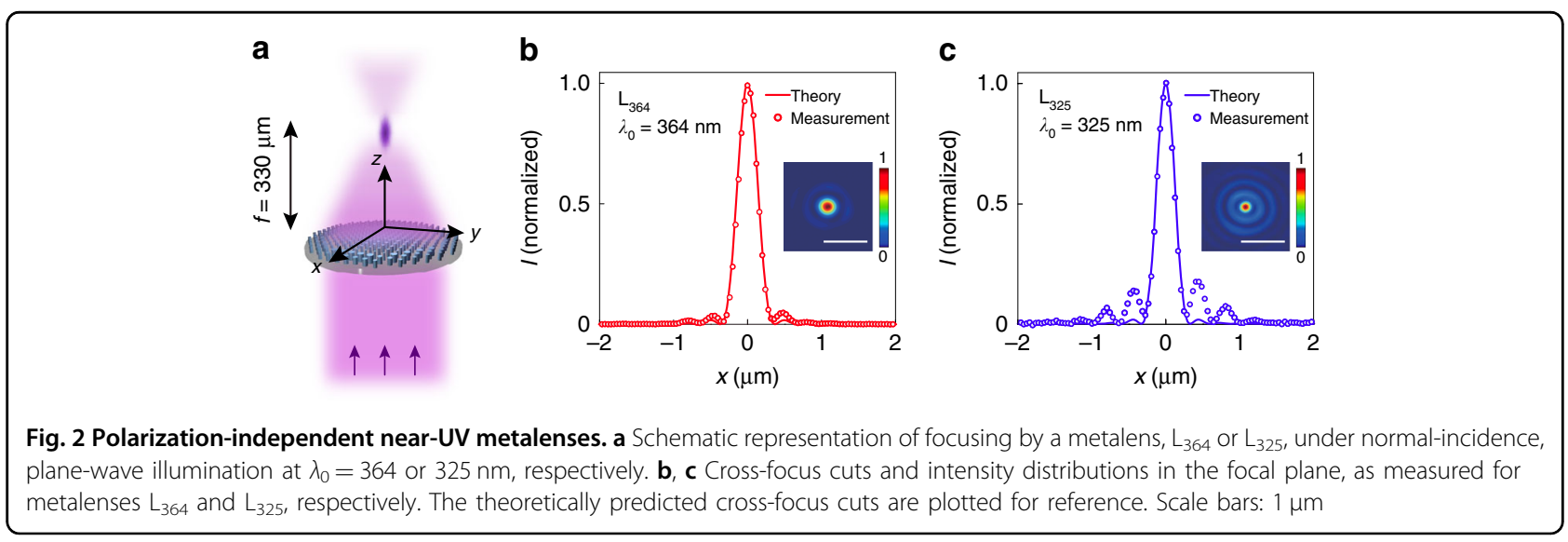



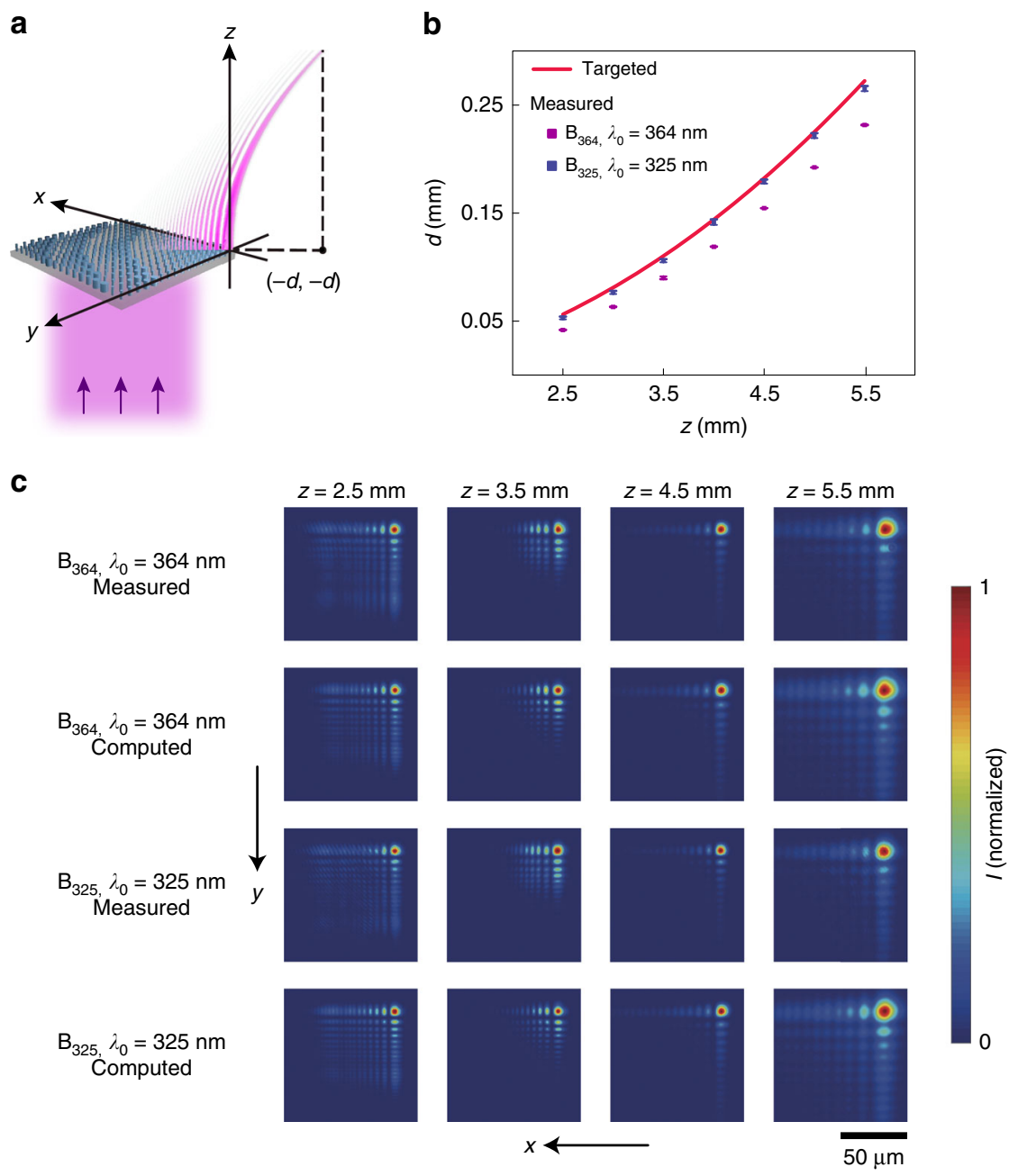

Fig. 3 Polarization-independent near-UV self-accelerating beam generators. a Schematic representation of the generation of a self-accelerating beam by a metasurface, $B_{364}$ or $B_{325}$, under normal-incidence, plane-wave illumination at $\lambda_{0}=364$ or $325 \mathrm{~nm}$, respectively. b Measured transverse deflection in different $z$ planes (ranging from 2.5 to $5.5 \mathrm{~mm}$, with an increment of $0.5 \mathrm{~mm}$ ) for the illumination of $B_{364}$ and $B_{325}$, respectively, at respective operation wavelengths $\lambda_{0}=364$ and $325 \mathrm{~nm}$. The error bars denote one standard deviation of the measured data. The targeted beam trajectory, $d=9 z^{2}$, is shown for reference. c Measured and computed $x y$-plane intensity distributions (normalized) at different $z$ planes for both devices at their designated wavelengths of operation. Each distribution is displayed over an equal square area with a side length of $120 \mu \mathrm{m}$ but shifted along the $-x y$ direction as a function of increasing $z$, such that the center of the main lobe maintains an invariant position within each image

aperture of NA $=0.6$ and given by the Airy disk function $I(x)=\left[2 J_{1}(A) / A\right]^{2}$, where $J_{1}$ is the Bessel function of the first kind of order one, and $A=2 \pi \mathrm{NA} x / \lambda_{0}$ (Fig. 2b, c). Metalens $\mathrm{L}_{325}$ exhibits a less-than-ideal focusing profile with larger side lobes, which could be due to fabrication imperfections and a nonideal realization of the required phase shift profile. The focusing efficiencies, defined as the ratio of the optical power of the focused spot to the total power illuminating the metalens, are $(55.17 \pm 2.56) \%\left(\mathrm{~L}_{364}\right)$ and $(56.28 \pm 1.37) \%\left(\mathrm{~L}_{325}\right)$. The cited uncertainties represent one standard deviation of the measured data.

Next, we demonstrate polarization-independent metasurfaces that can transform a normally incident plane wave into a diffraction-free output beam propagating along a curved trajectory, i.e., a self-accelerating beam $(\mathrm{SAB})^{31-33}$. Two $270-\mu \mathrm{m}$-square $\mathrm{SAB}$ generator designs, $B_{364}$ and $B_{325}$, are implemented for operation at the respective wavelengths of 364 and $325 \mathrm{~nm}$ (Fig. 3a). The SAB generator design and operation are conveniently described using a Cartesian coordinate system in which the constituent metasurface is located in the $z=0$ plane and the first $x y$ quadrant, with one corner positioned at the origin. The implemented SAB for each targeted freespace wavelength, $\lambda_{0}=364$ and $325 \mathrm{~nm}$, is characterized by an L-shaped wave-packet of the main lobe centered on the trajectory $y=x=-a z^{2}$, where $a=9 \mathrm{~m}^{-1}$ (in other words, originating from $(0,0,0)$ and propagating in the $+z$ direction in a curved trajectory confined to the plane 

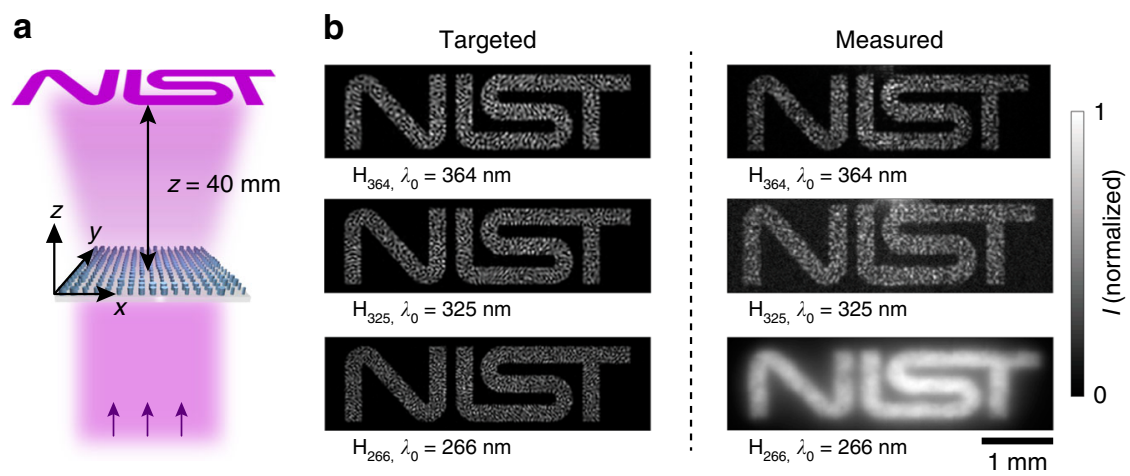

Fig. 4 Polarization-independent near-UV and deep-UV metaholograms. a Schematic representation of the holographic image projection by a metahologram, $\mathrm{H}_{364}, \mathrm{H}_{325}$, or $\mathrm{H}_{266}$, under normal-incidence, plane-wave illumination at $\lambda_{0}=364,325$, or $266 \mathrm{~nm}$, respectively. b Targeted (left panel) and measured (right panel) holographic images projected by the metaholograms $\mathrm{H}_{364}, \mathrm{H}_{325}$, and $\mathrm{H}_{266}$ in the $\mathrm{z}=40 \mathrm{~mm}$ plane

$y=x$ with a height above the surface given by $z=\sqrt{d / a}$, where $d=|x|=|y|$ is the lateral displacement). The targeted $\mathrm{SAB}$ can be generated by implementing a phase shift profile $\phi^{\mathrm{B}}\left(x, y, \lambda_{0}\right)=\bmod \left(-\frac{8 \pi}{3 \lambda_{0}} \sqrt{a}\left(x^{\frac{3}{2}}+y^{\frac{3}{2}}\right), 2 \pi\right)$ in the metasurface ${ }^{34}$. The measured lateral displacement values $d(z)$ are observed to closely match, in each case, the calculated values based on the targeted trajectory (Fig. 3b and Supplementary Information, Section X). The experimental SAB generated by each device exhibits diffractionfree characteristics with $x y$-plane intensity distributions similar to the intensity distributions numerically computed using the angular spectrum representation method $^{35}$, assuming an ideal metasurface realization with both the designed phase shift profile $\phi^{\mathrm{B}}$ and unity transmittance $T$ (Fig. 3c). The measured efficiencies, defined as the ratio of the total optical power of the SAB in the $z=$ $5 \mathrm{~mm}$ plane to the total power illuminating the metasurface, are $(46.75 \pm 2.31) \%\left(\mathrm{~B}_{364}\right)$ and $(67.42 \pm 4.43) \%\left(\mathrm{~B}_{325}\right)$. The efficiencies compare favorably to that of a recently reported $\mathrm{TiO}_{2}$-based self-accelerating beam generator operating at visible frequencies ${ }^{36}$.

As a final demonstration of polarization-independent UV metasurfaces, we demonstrate three metaholograms, denoted $\mathrm{H}_{364}, \mathrm{H}_{325}$, and $\mathrm{H}_{266}$, operating at three respective UV wavelengths $\lambda_{0}=364,325$, and $266 \mathrm{~nm}$ (Fig. 4a). Implementing computer-generated holograms with metasurfaces enables high-efficiency and low-noise operation, fine spatial resolution, a compact footprint, and multiplexing capability ${ }^{37-40}$. Each demonstrated metahologram, which occupies a square area with a side length of $270 \mu \mathrm{m}$, is mapped to a Cartesian coordinate system in which the constituent metasurface is located in the $z=0$ plane and the first $x y$ quadrant, with one corner positioned at the origin. The Gerchberg-Saxton algorithm $^{41}$ is employed to calculate the phase shift profiles, $\phi_{364}^{\mathrm{H}}\left(x, y, \lambda_{0}\right), \phi_{325}^{\mathrm{H}}\left(x, y, \lambda_{0}\right)$, and $\phi_{266}^{\mathrm{H}}\left(x, y, \lambda_{0}\right)$, required to project a holographic "NIST" image located in the $z=$
$40 \mathrm{~mm}$ plane, under normal-incidence, plane-wave illumination (Supplementary Information, Section XI). An additional offset of $y=-3 \mathrm{~mm}$ is added to avoid overlap of the generated holographic image with the residual directly transmitted beam. The images projected by metaholograms $\mathrm{H}_{364}, \mathrm{H}_{325}$, and $\mathrm{H}_{266}$ are measured (Supplementary Information, Sections XII and XIII) and displayed in the right panel of Fig. 4b. Each of the experimental holographic images faithfully replicates the shape of the corresponding target image (left panel of Fig. 4b), numerically computed assuming an ideal metahologram realization with both the designed phase shift profile $\phi^{\mathrm{H}}$ for a given operation wavelength and unity transmittance $T$. In addition, the speckle patterns filling the shapes of the measured images projected by metaholograms $\mathrm{H}_{364}$ and $\mathrm{H}_{325}$ exhibit numerous similarities with those of the corresponding target images; the as-measured holographic image projected by metahologram $\mathrm{H}_{366}$ does not offer the possibility of such a comparison due to the employed fluorescence transduction characterization scheme, which washes out the details of the speckle patterns. The measured efficiencies for metaholograms $\mathrm{H}_{364}$ and $\mathrm{H}_{325}$, defined as the ratio of the total optical power of the holographic image to the total power illuminating the structure, are $(62.99 \pm 4.14) \%$ and $(71.78 \pm 2.06) \%$, respectively. The measured efficiency for metahologram $\mathrm{H}_{266}$, defined as the ratio of the total fluorescence power of the holographic image to the fluorescence power of the light illuminating the structure (Supplementary Information, Section XIII), is $(60.67 \pm 2.60) \%$. These efficiency values are comparable to those of recently reported $\mathrm{TiO}_{2}$ based metaholograms operating in the visible range ${ }^{26}$.

\section{Spin-multiplexed UV metasurfaces}

Metasurfaces have been demonstrated to switch between distinct optical outputs, such as different holographic images or differently oriented beams, under the 


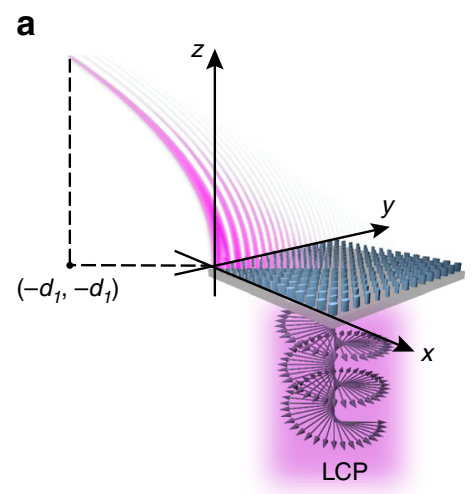

b

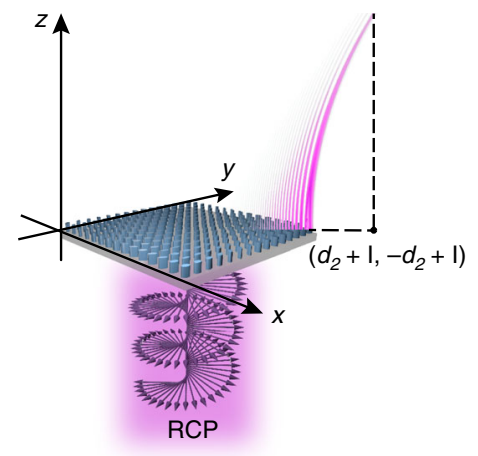

C

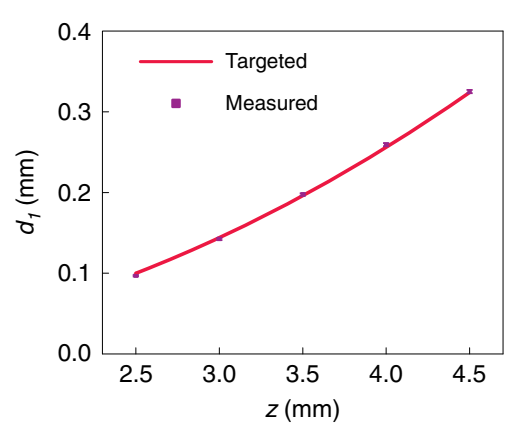

d

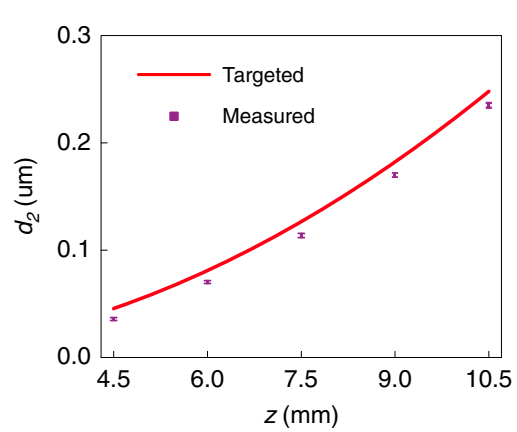

e

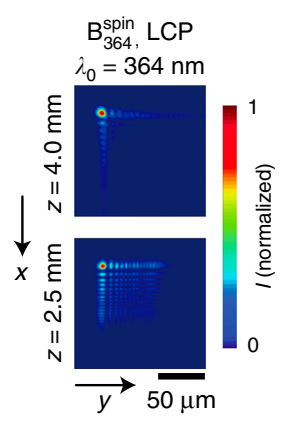

f

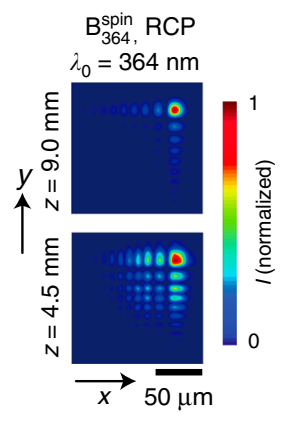

Fig. 5 Spin-multiplexed near-UV self-accelerating beam generator. $\mathbf{a}$, b Schematic representation of the generation of a self-accelerating beam by the spin-multiplexed metasurface, $B_{364}^{\text {spin }}$, under normal-incidence, plane-wave LCP a or RCP $\mathbf{b}$ illumination at $\lambda_{0}=364 \mathrm{~nm}$. $\mathbf{c}$ Measured transverse deflection in different $z$ planes (ranging from 2.5 to $4.5 \mathrm{~mm}$, with an increment of $0.5 \mathrm{~mm}$ ) for LCP illumination of $B_{364}^{\text {spin }}$ at its operation wavelength of $\lambda_{0}=364 \mathrm{~nm}$. The error bars denote one standard deviation of the measured data. The targeted beam trajectory, $d=16 z^{2}$, is shown for reference. $\mathbf{d}$ Measured transverse deflection in different $z$ planes (ranging from 4.5 to $10.5 \mathrm{~mm}$, with an increment of $1.5 \mathrm{~mm}$ ) for RCP illumination of $B_{364}^{\text {spin }}$ at its operation wavelength of $\lambda_{0}=364 \mathrm{~nm}$. The error bars denote one standard deviation of the measured data. The targeted beam trajectory, $d=2.25 z^{2}$, is shown for reference. $\mathbf{e}, \mathbf{f}$ Measured $x y$-plane intensity distributions (normalized) in different $z$ planes for the device at its designated wavelength of operation under either LCP illumination e or RCP illumination $\mathbf{f}$. Each distribution is displayed over an equal square area with a side length of $140 \mu \mathrm{m}$ but shifted along the $-x y \mathbf{e}$ or $x y \mathbf{f}$ direction as a function of increasing $z$, such that the center of the main lobe maintains an invariant position within each image

control of the fundamental optical state of the input beam, e.g., polarization ${ }^{42,43}$, or a spatial feature of the input beam such as the angle of incidence ${ }^{44}$. Here, we demonstrate, for the first time, spin-multiplexed UV metasurfaces that can switch between distinct outputs depending on the handedness of the input light (left-hand circularly polarized, LCP, or right-hand circularly polarized, RCP). The detailed design procedure is elaborated in Supplementary Information, Section XIV.

As a first demonstration of a polarization-dependent, spin-multiplexed UV metasurface, we implement a selfaccelerating beam generator operating at $\lambda_{0}=364 \mathrm{~nm}$, denoted as $\mathrm{B}_{364}^{\mathrm{spin}}$, that generates SABs following different trajectories under the control of the handedness of circularly polarized incident light. The spin-multiplexed SAB generator, which occupies a square area with a side length of $l=330 \mu \mathrm{m}$, is referenced to a Cartesian coordinate system in which the constituent metasurface is located in the $z=0$ plane and the first $x y$ quadrant, with one corner positioned at the origin. Two distinct phase shift profiles, $\phi^{\mathrm{LCP}}\left(x, y, \lambda_{0}\right)=$ $\bmod \left(-\frac{8 \pi}{3 \lambda_{0}} \sqrt{16}\left(x^{\frac{3}{2}}+y^{\frac{3}{2}}\right), 2 \pi\right) \quad$ and $\quad \phi^{\mathrm{RCP}}\left(x, y, \lambda_{0}\right)=$ $\bmod \left(-\frac{8 \pi}{3 \lambda_{0}} \sqrt{2.25}\left((l-x)^{\frac{3}{2}}+(l-y)^{\frac{3}{2}}\right), 2 \pi\right)$, are targeted for the device operation to yield SABs exiting the metasurface from opposite corners and following different trajectories, $y=x=-d_{1}=-16 z^{2}$ and $(y-l)=(x-l)=d_{2}=$ $2.25 z^{2}$, under LCP and RCP illuminations, respectively (Fig. $5 \mathrm{a}, \mathrm{d})$. The measured lateral displacement values, $d_{1}(z)$ and $d_{2}(z)$, are observed to closely match, in each case, the calculated values based on the targeted trajectory (Fig. 5c, d). The experimental $\mathrm{SAB}$ generated by the device exhibits 
a

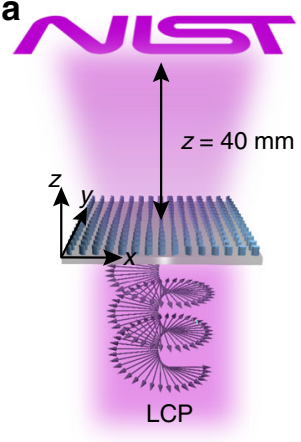

C

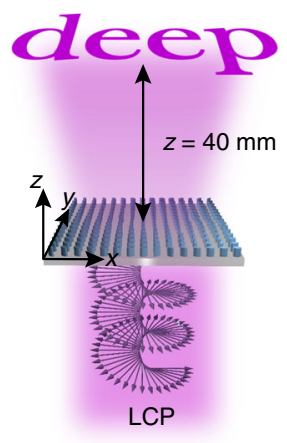

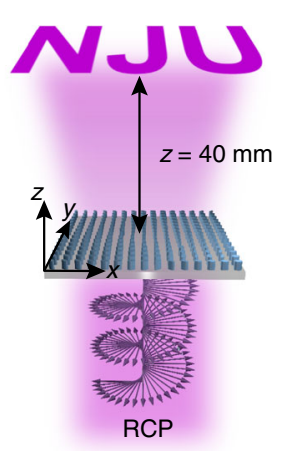

b
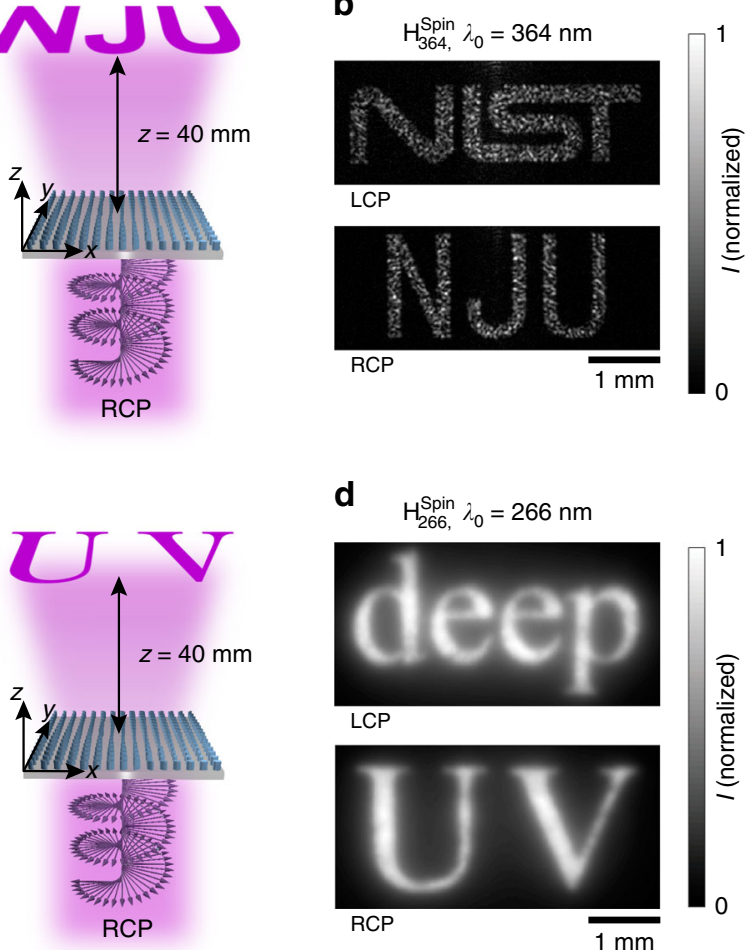

d

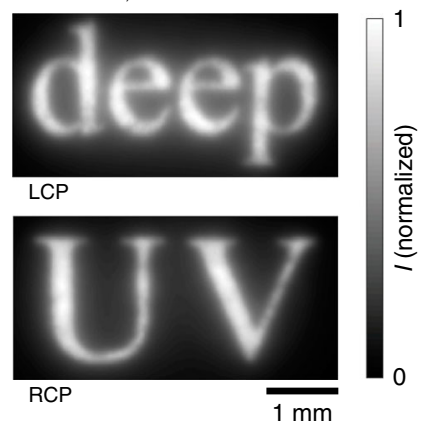

Fig. 6 Spin-multiplexed near- and deep-UV metaholograms. a Schematic representation of the holographic image projection by the spinmultiplexed metahologram $\mathrm{H}_{364}^{\text {spin }}$ under LCP or RCP illumination at $\lambda_{0}=364 \mathrm{~nm}$. b Measured holographic images projected by metahologram $\mathrm{H}_{364}^{\text {spin }}$ in the $z=40 \mathrm{~mm}$ plane under LCP illumination (top image) and RCP illumination (bottom image). $\mathbf{c}$ Schematic representation of the holographic image projection by the spin-multiplexed metahologram $H_{266}^{\text {spin }}$ under LCP or RCP illumination at $\lambda_{0}=266 \mathrm{~nm}$. $\mathbf{d}$ Measured holographic images projected by metahologram $H_{266}^{\text {spin }}$ in the $z=40 \mathrm{~mm}$ plane under LCP illumination (top image) and RCP illumination (bottom image)

diffraction-free characteristics with $x y$-plane intensity distributions (Fig. 5e, f) similar to the targeted intensity distributions (Supplementary Information, Figs. S14 and S15), numerically computed assuming an ideal metasurface realization with both the designed phase shift profile $\phi^{\mathrm{LCP}}\left(\phi^{\mathrm{RCP}}\right)$ and unity transmittance $T$. The measured efficiency under LCP illumination (RCP illumination), defined as the ratio of the total optical power of the SAB in the $z=4.5 \mathrm{~mm}[z=$ $10.5 \mathrm{~mm}$ ] plane to the total power illuminating the metasurface, is $(38.42 \pm 1.95) \%[(61.90 \pm 2.03) \%]$. The reduced efficiency under LCP illumination, compared to the RCP case, can be attributed to challenges associated with implementing a phase shift profile of a higher spatial gradient (Supplementary Information, Section XVI).

Next, we demonstrate a spin-controlled metahologram operating at the same near-UV wavelength of $364 \mathrm{~nm}$. The 330- $\mu \mathrm{m}$-square metahologram, $\mathrm{H}_{364}^{\text {spin }}$, located in the $z=0$ plane, is designed to project a holographic "NIST" image (for LCP illuminating light) and "NJU" image (for RCP illuminating light) at $\lambda_{0}=364 \mathrm{~nm}$, all located in the $x y$-plane at $z=40 \mathrm{~mm}$ with an offset of $y=-3 \mathrm{~mm}$ (Fig. 6a; the corresponding phase shift profiles are plotted in the Supplementary Information, Fig. S17). Both of the experimentally captured holographic images (Fig. 6b) faithfully replicate the shape of the corresponding targeted image computed from the designed phase profiles, including some fine grain details (Supplementary Information, Fig. S18). The measured efficiencies, defined as the ratio of the total optical power of the holographic image to the total power illuminating the metahologram, are $(54.02 \pm 2.22) \%$ (under LCP illumination) and (53.76 \pm 2.42)\% (under RCP illumination), respectively.

Finally, a spin-multiplexed metahologram, $\mathrm{H}_{266}^{\text {spin }}$, occupying a square area with a side length of $320 \mu \mathrm{m}$, is implemented for operation at the deep-UV wavelength of $266 \mathrm{~nm}$. The device, located in the $z=0$ plane, is designed to project, at $\lambda_{0}=266 \mathrm{~nm}$, a holographic "deep" image for LCP illumination and a holographic "UV" image for RCP illumination, where both images are located in the $z=$ $40 \mathrm{~mm}$ plane with a lateral offset of $y=-3 \mathrm{~mm}$ (Fig. 6c; the corresponding phase shift profiles are plotted in the Supplementary Information, Fig. S19). Each of the experimental holographic images (Fig. 6d) faithfully replicates the shape of the corresponding target image (Supplementary Information, Fig. S20), including subtle details of the chosen font, such as the linewidth variation 
and serif. The measured efficiencies, defined as the ratio of the total fluorescence power of the holographic image to the fluorescence power of light illuminating the structure, are $(58.95 \pm 1.95) \%$ under LCP illumination and $(61.23 \pm 1.49) \%$ under RCP illumination.

\section{Discussion}

Given the negligible extinction coefficient of the lowtemperature-ALD deposited $\mathrm{HfO}_{2}$ down to its bandgap $\left(\lambda_{0} \approx 217 \mathrm{~nm}\right)$ and the high patterning fidelity of the Damascene process, it should be straightforward to push the metasurface operation wavelengths to significantly shorter values than those demonstrated here. In addition, an experimental demonstration of a broader range of device functionalities in the deep-UV regime other than hologram projection should be possible by using a continuous-wave light source and an appropriate direct imaging system. Moreover, the efficiency of $\mathrm{HfO}_{2}$-based metasurface devices can be improved by further optimizing the Damascene process or by employing advanced metasurface design strategies, such as topology optimization $^{45}$ and the generalized Huygens principle ${ }^{46,47}$.

In conclusion, an assortment of high-performance metasurface components operating in the UV regime, including wavelengths down to the record-short deep-UV range, is demonstrated by using $\mathrm{HfO}_{2}$, a CMOS-compatible, widebandgap, and low-loss dielectric material, and an associated fabrication process based on low-temperature ALD and Damascene lithography. This approach paves the way towards further development of "flat" UV optical elements with customized functionalities and their integration into chip-scale nanophotonic systems, enabling applications such as atom trapping, fluorescence imaging, and circular dichroism spectroscopy with a compact form factor.

\section{Materials and methods \\ Metasurface fabrication process}

As the first step in the metasurface fabrication process, 500- $\mu \mathrm{m}$-thick, double-side-polished UV-grade fused silica wafers are vapor-coated $\left(150^{\circ} \mathrm{C}\right)$ with an adhesion-enhancing monolayer of hexamethyldisilizane (HMDS). A layer of ZEP 520A resist is spin-coated onto the substrate, followed by baking on a hot plate at $180^{\circ} \mathrm{C}$ for $10 \mathrm{~min}$. The spin speed is adjusted to yield a resist thickness varying between 400 and $550 \mathrm{~nm}$ (as characterized by spectroscopic ellipsometry), depending on the specific metasurface design. To suppress charging during electron beam (e-beam) lithography, a 20-nmthick $\mathrm{Al}$ layer is thermally evaporated onto the ZEP layer (deposition rate of $0.1 \mathrm{~nm} / \mathrm{s}$ ). The ZEP-resist template is fabricated using e-beam lithography (accelerating voltage of $100 \mathrm{kV}$ and beam current of $0.2 \mathrm{nA}$ ), followed by Al layer removal (AZ $400 \mathrm{~K} \mathrm{1:3} \mathrm{developer} \mathrm{for} 2 \mathrm{~min}$ and DI water for $1 \mathrm{~min}$ ) and resist development (hexyl acetate for $2 \mathrm{~min}$ and isopropyl alcohol for $30 \mathrm{~s}$ ). The deposition of $\mathrm{HfO}_{2}$ (deposition rate: $0.11 \mathrm{~nm} /$ cycle) is then performed using the low-temperature ALD described below. For all of the processed structures, the deposition thickness is chosen to be $200 \mathrm{~nm}$, which not only exceeds the largest radius (or the largest semiminor axis length) of the circular (or elliptical) openings of the exposed resist patterns for all metasurface designs, providing complete filling of the patterns, but also provides a substantial over-coating of the resist, yielding a quasi-planar top surface (Supplementary Information, Section VI). Following the ALD, the $\mathrm{HfO}_{2}$ layer is back-etched to the resist top surface using argon (Ar) ion milling ( $\mathrm{HfO}_{2}$ mill rate of $\approx 0.4 \mathrm{~nm} / \mathrm{s}$ ). During the Ar ion milling, a non-patterned, planar $\mathrm{HfO}_{2}$ sample of the same initial thickness is also back-etched, and its film thickness is periodically monitored by spectroscopic ellipsometry to ensure that a proper milling time is employed. Finally, the remaining resist is removed by soaking in a solvent, yielding circular or elliptical $\mathrm{HfO}_{2}$ posts with smooth and straight sidewall profiles (due to the resist templating process), heights varying from 400 to $550 \mathrm{~nm}$ (depending on the specific metasurface), and aspect ratios varying from $\approx 3$ to $\approx 11$.

\section{Low-temperature TDMAH-based $\mathrm{HfO}_{2}$ ALD}

In step 1 of the ALD cycle, TDMAH vapor ( $\mathrm{Hf}$ $\left.\left[\left(\mathrm{CH}_{3}\right)_{2} \mathrm{~N}\right]_{4}\right)$ is pulsed into the ALD chamber for a duration of $t_{1}=1 \mathrm{~s}$, reacting with the dangling $\mathrm{O}-\mathrm{H}$ bonds on the hafnium-coated surface to create a new solid monolayer of $\mathrm{Hf}\left[\left(\mathrm{CH}_{3}\right)_{2} \mathrm{~N}\right]_{2} \mathrm{O}$ and generate the gas by-product $\left(\mathrm{CH}_{3}\right)_{2} \mathrm{NH}$ (dimethylamine). In step 2, high-purity nitrogen $\left(\mathrm{N}_{2}\right)$ gas is flowed for a duration of $t_{2}=75 \mathrm{~s}$ to fully remove any un-reacted TDMAH vapor and dimethylamine by-product from the chamber. In step 3 , water vapor is pulsed into the chamber for a duration of $t_{3}=60 \mathrm{~ms}$, reacting with the $\mathrm{Hf}\left[\left(\mathrm{CH}_{3}\right)_{2} \mathrm{~N}\right]_{2} \mathrm{O}$ to create a monolayer of $\mathrm{HfO}_{2}$ on the surface. Finally, in step 4, the excessive water vapor and the dimethylamine reaction by-product are completely removed from the chamber by $\mathrm{N}_{2}$ purging for a duration of $t_{4}=75 \mathrm{~s}$.

\section{Acknowledgements}

Q.F., Y.L., and T.X. acknowledge support from The National Key R\&D Program of China (Grant Nos. 2017 YFA0303700 and 2016YFA0202100) and the National Science Foundation of China (Grant No. 11774163). C.Z., S.D., W.Z., and A.A. acknowledge support under the Cooperative Research Agreement between the University of Maryland and the National Institute of Standards and Technology (NIST) Physical Measurement Laboratory, Award No. 70NANB14H209, through the University of Maryland. C.Z. acknowledges start-up funding from Huazhong University of Science and Technology and helpful discussions with Dr. Huazhi Li. Certain commercial equipment and software are identified in this documentation to describe the subject adequately. Such identification does not imply recommendation or endorsement by the NIST, nor does it imply that the equipment identified is necessarily the best available for the purpose. 


\section{Author details}

'School of Optical and Electronic Information \& Wuhan National Laboratory for Optoelectronics, Huazhong University of Science and Technology, 430074 Wuhan, China. ${ }^{2}$ Physical Measurement Laboratory, National Institute of Standards and Technology, 20899 Gaithersburg, MD, USA. ${ }^{3}$ Maryland Nanocenter, University of Maryland, College Park, MD 20742, USA. ${ }^{4}$ National Laboratory of Solid State Microstructures, College of Engineering and Applied Sciences and Collaborative Innovation Center of Advanced Microstructures, Nanjing University, 210093 Nanjing, China

\section{Author contributions}

The project was initiated by C.Z. The device fabrication and characterization were performed by C.Z., S.D., W.Z., and A.A. The simulations were performed by Q.F., S.D., C.Z. with further analysis by Y.L., T.X., and H.J.L. All authors contributed to the interpretation of results and participated in the manuscript preparation.

\section{Conflict of interest}

The authors declare that they have no conflict of interest.

Supplementary information is available for this paper at https://doi.org/ 10.1038/s41377-020-0287-y.

Received: 1 December 2019 Revised: 25 February 2020 Accepted: 11 March 2020

Published online: 09 April 2020

\section{References}

1. Yu, N. F. \& Capasso, F. Flat optics with designer metasurfaces. Nat. Mater. 13, 139-150 (2014).

2. Hsiao, H. H., Chu, C. H. \& Tsai, D. P. Fundamentals and applications of metasurfaces. Small Methods 1, 1600064 (2017).

3. Kildishev, A. V., Boltasseva, A. \& Shalaev, V. M. Planar photonics with metasurfaces. Science 339, 1232009 (2013).

4. Ding, F., Pors, A. \& Bozhevolnyi, S. I. Gradient metasurfaces: a review of fundamentals and applications. Rep. Prog. Phys. 81, 026401 (2018).

5. Chen, H. T., Taylor, A. J. \& Yu, N. F. A review of metasurfaces: physics and applications. Rep. Prog. Phys. 79, 076401 (2016).

6. Kamali, S. M. et al. A review of dielectric optical metasurfaces for wavefront control. Nanophotonics 7, 1041-1068 (2018).

7. Zhang, L. et al. Advances in full control of electromagnetic waves with metasurfaces. Adv. Opt. Mater. 4, 818-833 (2016).

8. Sun, S. L. et al. Electromagnetic metasurfaces: physics and applications. Adv. Opt. Photonics 11, 380-479 (2019).

9. Luo, X. G. Subwavelength optical engineering with metasurface waves. Adv. Opt. Mater. 6, 1701201 (2018).

10. Arbabi, A. et al. Dielectric metasurfaces for complete control of phase and polarization with subwavelength spatial resolution and high transmission. Nat. Nanotechnol. 10, 937-943 (2015).

11. Lin, D. M. et al. Dielectric gradient metasurface optical elements. Science $\mathbf{3 4 5}$ 298-302 (2014).

12. Divitt, $\mathrm{S}$. et al. Ultrafast optical pulse shaping using dielectric metasurfaces. Science 364, 890-894 (2019).

13. Khorasaninejad, M. et al. Metalenses at visible wavelengths: diffraction-limited focusing and subwavelength resolution imaging. Science 352, 1190-1194 (2016).

14. Chen, W. T. et al. A broadband achromatic metalens for focusing and imaging in the visible. Nat. Nanotechnol. 13, 220-226 (2018).

15. Wang, S. M. et al. A broadband achromatic metalens in the visible. Nat. Nanotechnol. 13, 227-232 (2018).

16. Ren, H. R. et al. Metasurface orbital angular momentum holography. Nat. Commun. 10, 2986 (2019).

17. Day, R. N. \& Davidson, M. W. The fluorescent protein palette: tools for cellular imaging. Chem. Soc. Rev. 38, 2887-2921 (2009).
18. Greenfield, N. J. Using circular dichroism spectra to estimate protein secondary structure. Nat. Protoc. 1, 2876-2890 (2006).

19. Ludlow, A. D. et al. Optical atomic clocks. Rev. Mod. Phys. 87, 637-701 (2015).

20. Huang, $K$. et al. Ultraviolet metasurfaces of $\approx 80 \%$ efficiency with antiferromagnetic resonances for optical vectorial anti-counterfeiting. Laser Photonics Rev. 13, 1800289 (2019).

21. Deng, Y. et al. All-silicon broadband ultraviolet metasurfaces. Adv. Mater. 30, 1802632 (2018).

22. Robertson, J. High dielectric constant oxides. Eur. Phys. J. Appl. Phys. 28, 265-291 (2004).

23. Wilk, G. D., Wallace, R. M. \& Anthony, J. M. High-k gate dielectrics: current status and materials properties considerations. J. Appl. Phys. 89, 5243-5275 (2001).

24. Andricacos, P. C. et al. Damascene copper electroplating for chip interconnections. IBM J. Res. Dev. 42, 567-574 (1998).

25. Schmid, G. M. et al. Implementation of an imprint damascene process for interconnect fabrication. J. Vac. Sci. Technol. B 24, 1283-1291 (2006).

26. Devlin, R. C. et al. Broadband high-efficiency dielectric metasurfaces for the visible spectrum. Proc. Natl Acad. Sci. USA 113, 10473-10478 (2016).

27. Lowalekar, V. \& Raghavan, S. Etching of zirconium oxide, hafnium oxide, and hafnium silicates in dilute hydrofluoric acid solutions. J. Mater. Res. 19 1149-1156 (2011).

28. Liu, X. Y. et al. ALD of hafnium oxide thin films from tetrakis(ethylmethylamino) hafnium and ozone. J. Electrochem. Soc. 152, G213-G219 (2005).

29. Kukli, K. et al. Comparison of hafnium oxide films grown by atomic layer deposition from iodide and chloride precursors. Thin Solid Films 416, 72-79 (2002).

30. Hausmann, D. M. et al. Atomic layer deposition of hafnium and zirconium oxides using metal amide precursors. Chem. Mater. 14, 4350-4358 (2002).

31. Siviloglou, G. A. et al. Observation of accelerating airy beams. Phys. Rev. Lett. 99, 213901 (2007).

32. Siviloglou, G. A. \& Christodoulides, D. N. Accelerating finite energy airy beams Opt. Lett. 32, 979-981 (2007).

33. Henstridge, M. et al. Accelerating light with metasurfaces. Optica 5, 678-681 (2018).

34. Cottrell, D. M., Davis, J. A. \& Hazard, T. M. Direct generation of accelerating airy beams using a 3/2 phase-only pattern. Opt. Lett. 34, 2634-2636 (2009).

35. Novotny, L. \& Hecht, B. Principles of Nano-Optics. (Cambridge University Press, Cambridge, 2006)

36. Fan, Q. B. et al. Broadband generation of photonic spin-controlled arbitrary accelerating light beams in the visible. Nano Lett. 19, 1158-1165 (2019).

37. Wang, L. et al. Grayscale transparent metasurface holograms. Optica 3, 1504-1505 (2016).

38. Wang, B. et al. Polarization-controlled color-tunable holograms with dielectric metasurfaces. Optica 4, 1368-1371 (2017).

39. Yue, Z. et al. Nanometric holograms based on a topological insulator material. Nat. Commun. 8, 15354 (2017)

40. Huang, L. L., Zhang, S. \& Zentgraf, T. Metasurface holography: from fundamentals to applications. Nanophotonics 7, 1169-1190 (2018).

41. Gerchberg, R. W. \& Saxton, W. O. A practical algorithm for the determination of phase from image and diffraction plane pictures. Optik $\mathbf{3 5}$, 237-246 (1972)

42. Mueller, J. P. B. et al. Metasurface polarization optics: independent phase control of arbitrary orthogonal states of polarization. Phys. Rev. Lett. 118, 113901 (2017).

43. Wang, K. et al. Quantum metasurface for multiphoton interference and state reconstruction. Science 361, 1104-1108 (2018).

44. Kamali, S. M. et al. Angle-multiplexed metasurfaces: encoding independent wavefronts in a single metasurface under different illumination angles. Phys. Rev. X 7, 041056 (2017).

45. Phan, T. et al. High-efficiency, large-area, topology-optimized metasurfaces. Light: Sci. Appl. 8, 48 (2019).

46. Decker, M. et al. High-efficiency dielectric Huygens' surfaces. Adv. Opt. Mater. 3, 813-820 (2015).

47. Pfeiffer, C. \& Grbic, A. Metamaterial Huygens' surfaces: tailoring wave fronts with reflectionless sheets. Phys. Rev. Lett. 110, 197401 (2013). 